\title{
Ciencias Químicas
}

Citación: Chalarca-Vélez JR, Gaviria D. Modelamiento por homología in silico de la quinoproteína glucosa deshidrogenasa unida a membrana en Pseudomonas fluorescens. Rev. Acad. Colomb. Cienc. Ex. Fis. Nat. 44(173):1099-1112, octubre-diciembre de 2020. doi: https://doi.org/10.18257/ raccefyn. 1154

Editor: Luis Fernando Echeverri

\section{*Correspondencia:}

Julia Rosa Chalarca V;

juliar-chalarcav@unilibre.edu.co

Recibido: 11 de marzo de 2020 Aceptado: 30 de septiembre de 2020

Publicado: 5 diciembre de 2020

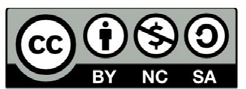

Este artículo está bajo una licencia de Creative Commons ReconocimientoNoComercial-Compartir Igual 4.0 Internacional
Artículo original

\section{Modelamiento por homología in silico de la quinoproteína glucosa deshidrogenasa unida a membrana en Pseudomonas fluorescens}

\author{
In silico modeling by homology of membrane-bound \\ quinoprotein glucose dehydrogenase in Pseudomonas \\ fluorescens
}

\author{
(D) Julia Rosa Chalarca-Vélez*, Duverney Gaviria \\ Programa de Microbiología, Universidad Libre, Pereira, Colombia
}

\section{Resumen}

Entre las aplicaciones del modelamiento por homología están la predicción de ligandos, el análisis de mutaciones, y las actividades catalíticas. Normalmente, el uso de varios moldes produce modelos más precisos en la medida en que capturan mejor las variantes estructurales entre todas las posibles conformaciones de las proteínas de una familia. El modelamiento comparativo es una estrategia útil cuando no se tiene información en las bases de datos y se quiere hacer un ensayo experimental relacionado con la estructura y el funcionamiento de una proteína. En el presente trabajo se analizó estructural y funcionalmente la quinoproteína glucosa deshidrogenasa unida a la membrana (membrane-bound PQQ-dependent glucose dehydrogenase, $m G D H$, PQQ mGDH) en Pseudomonas fluorescens, reconocida como un microorganismo promotor del crecimiento vegetal y una de cuyas funciones es la solubilización de fosfatos inorgánicos catalizados mediante PQQ mGDH. Esta es la primera enzima que participa en la oxidación directa de la glucosa transformando la D-glucosa a D-gluconato, proceso que es el inicio en la producción de ácidos orgánicos implicados en la solubilización de fosfatos inorgánicos. A pesar de la gran cantidad de estudios con esta enzima, no se ha reportado la determinación de su estructura tridimensional de la enzima quinoproteína glucosa deshidrogenasa (PQQ-GDH) en la bacteria P. fluorescens. En este estudio se comparó la secuencia de la proteína con las reportadas en las bases de datos para otras especies del género Pseudomonas y se propuso un modelo tridimensional de la enzima PQQ $\mathrm{mGDH}$ en $P$. fluorescens mediante el modelamiento por homología para caracterizar su estructura secundaria, el dominio catalítico, y el sitio de unión al cofactor pirroloquinolina quinona (PQQ), así como los dominios funcionales presentes en la proteína.

Palabras clave: Bioinformática; Modelamiento por homología; Quinoproteina.

\section{Abstract}

Homology modeling is used for ligand prediction, mutation analysis, and catalytic activities. The use of several templates produces more precise models to better capture the structural variants among all the possible conformations of the proteins in a family. Comparative modeling is a useful strategy when there is no information reported in databases and there is a need for an experimental trial related to the structure and functioning of a protein. In the present study, we analyzed the structure and function of the membrane-bound quinoprotein glucose dehydrogenase (PQQ mGDH) in Pseudomonas fluorescens, known to be a plant-growth-promoting microorganism, one of whose functions is the solubilization of inorganic phosphates catalyzed by the PQQ mGDH, which is the first enzyme that participates in the direct oxidation of glucose by transforming D-glucose to D-gluconate. This process is the beginning of the production of organic acids involved in the solubilization of inorganic phosphates. Despite the large number of studies on this enzyme, none determines the three-dimensional structure of the PQQ-GDH enzyme in P. fluorescens. In our study, we compared the protein sequence with those reported in the databases for other species of 
Pseudomonas using a three-dimensional model of PQQ mGDH in P. fluorescens based on homology modeling to characterize the secondary structure of the protein, the catalytic domain, the binding site for the cofactor pyrroloquinoline quinone (PQQ), as well as the functional domains present in this protein.

Keywords: Bioinformatics; Homology modelling; Quinoprotein.

\section{Introducción}

El fósforo (P) es uno de los elementos esenciales en el desarrollo y crecimiento de las plantas y representa aproximadamente el 0,2 \% del peso seco de una planta. Se ha establecido que, después del nitrógeno, es el segundo entre los nutrientes minerales que comúnmente limitan el crecimiento de los cultivos (Alori, et al., 2017). Las plantas adquieren el fósforo del suelo como ortofosfato inorgánico $(\mathrm{Pi})$, cuya concentración es controlada por procesos químicos y biológicos que lo fijan y liberan a través de complejas interacciones entre el suelo, los microorganismos que en él viven y las raíces de las plantas (Lidbury, et al., 2016). La solubilización de fosfato mineral es una actividad esencial de muchas rizobacterias con la cual promueven el crecimiento vegetal; entre los géneros mejor estudiados están Pseudomonas, Bacillus, Rhizobium, Micrococcus, Acinetobacter, Flavobacterium, Achromobacter, Erwinia y Agrobacterium. El mecanismo de su funcionamiento mejor caracterizado es la liberación de ácidos orgánicos en el espacio extracelular para retener los cationes divalentes (por ejemplo, $\mathrm{Ca}^{2+}$ ) a partir de las formas de fosfato mineral poco solubles, como la hidroxiapatita o el fosfato tricálcico, y, así, liberar el fosforo en una forma adecuada para la absorción por parte de la planta (An \& Moe, 2016).

Hasta ahora, las bacterias que solubilizan el fósforo (PSB) de forma más eficientes reportadas son las Gram negativas, las cuales utilizan la oxidación directa de la glucosa para producir ácido glucónico (GA) (Figura 1S, https:/www.raccefyn.co/index.php/ raccefyn/article/view/1154/2924).

Hasta la fecha se han identificado dos tipos de quinoproteína glucosa deshidrogenasa (PQQ-GDH) (EC 1.1.5.2): una unida a la membrana celular interna (mGDH) y la otra presente en forma soluble (sGDH), las cuales muestran actividad en el periplasma de bacterias Gram negativas. La mGDH se ha encontrado en muchas bacterias Gram negativas, como las Gluconobacter, Pseudomonas y Acinetobacter, en tanto que la sGDH es menos común y se ha reportado solo en $A$. calcoaceticus (An \& Moe, 2016). La conversión de glucosa en GA es catalizada por una glucosa deshidrogenasa unida a la membrana periplásmica (mGDH) que forma un complejo con el cofactor pirroloquinolina quinona (PQQ) (Ben Farhat, et al., 2013) codificado, a su vez, por un operón que en P. fluorescens consta de 11 genes (Krishnaraj \& Goldstein, 2002; Goldstein, et al., 2003). La PQQ es un compuesto que es sintetizado en fase estacionaria y funciona como un cofactor termoestable y soluble en agua. Se detectó inicialmente en bacterias metilotróficas y pertenece a la familia de cofactores del tipo o-quinona; las enzimas a las que se unen este tipo de cofactores se han designada como quinoproteínas porque poseen un sitio de unión para alguno de los miembros de la familia o-quinona. La PQQ es el único cofactor de esta familia que se une de manera no covalente a las enzimas como glucosa, metanol, sorbitol y glicerol deshidrogenasas (Vera-Cardoso, et al., 2017).

Se ha establecido que $P$. fluorescens tiene una gran capacidad para solubilizar el fósforo, lo que sucede a través de dos vías: la producción de ácidos orgánicos (cítrico, oxálico, glucónico) que modifican el pH del suelo favoreciendo la solubilización del fósforo inorgánico y liberando el fosfato al suelo a través de las fosfatasas, enzimas hidrolasas (monoesterasas y diesterasas fosfóricas) que actúan sobre las uniones ésteres liberando los grupos fosfatos de la materia orgánica. Ambas vías generan la mayor cantidad del fosfato disponible para ser absorbido por las raíces de las plantas (Pérez, et al., 2015). Otro aspecto destacable en $P$. fluorescens es la producción de sustancias estimuladoras del crecimiento, entre ellas las hormonas (auxinas, giberelinas y citoquininas); además, también producen aminoácidos específicos para el crecimiento vegetal. Su producción es posible siempre que se adecúe a la concentración de organismos en el sistema radicular y que en el suelo haya suficiente cantidad de materia orgánica (Pérez, et al., 2015). 
Uno de los principales objetivos de la biología estructural y de la química computacional es conocer con exactitud la estructura de las proteínas (Babnigg \& Joachimiak, 2010; Hamilton \& Huber, 2008). Para ello se recurre a métodos proteómicos que facilitan el aislamiento, purificación y cristalización de las proteínas, y al análisis mediante métodos de difracción de rayos X o de resonancia magnética nuclear (RMN) (Asahi, et al., 2010; Sánchez-Garcés, et al., 2012). Sin embargo, hay algunas dificultades para obtener proteínas cristalizadas en cantidades suficientemente grandes y de buena calidad para los posibles ensayos de difracción de rayos X (Chapman, et al., 2011). En consecuencia, todavía no se ha determinado la estructura tridimensional de la mayoría de las proteínas (Yamamoto, et al., 2010), lo que brinda la oportunidad para ensayar diferentes métodos que permitan conocer las estructuras de las proteínas de interés biológico para aplicarlas en procesos de agroecología. En los últimos años se ha avanzado notablemente en los métodos de cálculo de la química computacional orientados al modelamiento y evaluación de las proteínas, con lo que se han podido analizar in silico sus interacciones como alternativa viable para ahorrar tiempo en el diseño de experimentos con mejores expectativas de éxito y disminuir costos en materiales y reactivos (Wen, et al., 2011). El término "modelamiento por homología", también conocido como comparativo o basado en plantilla (templatebased modelling, TBM), consiste en modelar la estructura en 3D de una proteína utilizando a manera de molde otra estructura ya determinada experimentalmente. La estructura de la proteína es de gran ayuda en el estudio de su función, su dinámica, las interacciones con los ligandos y con otras proteínas.

En el presente estudio se propuso una estrategia para modelar y analizar mediante métodos bioinformáticos la estructura tridimensional de la enzima PQQ- mGDH en $P$. fluorescens caracterizando su estructura secundaria e identificando sus dominios funcionales y estructurales, así como su interacción con el cofactor PQQ.

\section{Metodología}

\section{Búsqueda de la secuencia de la proteína PQQ- mGDH en Pseudomonas fluorescens}

Se usó la plataforma del NCBI (Protein) (https://www.ncbi.nlm.nih.gov/protein/?term=) para buscar la proteína de interés, obtener su secuencia y, posteriormente, hacer el modelamiento por homología. Para ello, se tomó como referencia el genoma de la bacteria $P$. fluorescens F113, y se determinó en esta la secuencia correspondiente a la enzima PQQ- mGDH.

\section{Alineamiento por pares y múltiple}

Con la secuencia ya identificada, se hizo un alineamiento apareado con la herramienta BLAST (https://blast.ncbi.nlm.nih.gov/Blast.cgi) para detectar secuencias homólogas. Se extrajeron las secuencias correspondientes a otras especies del género y con estos datos se hizo un alineamiento múltiple con la aplicación del programa Clustal Omega (https:// www.ebi.ac.uk/Tools/msa/clustalo/) (Madeira, et al., 2019), con el fin de establecer el grado de diversidad e inferir si la estructura que se pretendía modelar podría aplicarse a las otras especies.

Modelamiento de la estructura tridimensional de la PQQ- mGDH de Pseudomonas fluorescens

A partir de la secuencia proteica de la PQQ- mGDH de $P$. fluorescens se hizo el modelamiento por homología con la aplicación de acceso libre Geno3D (https:/geno3dprabi. ibcp.fr/cgibin/geno3d_automat.pl?page=/GENO3D/geno3d_home.html) (Combet, et al., 2002). Para la visualización de todas las estructuras tridimensionales se utilizó el programa RasMol (Sayle, 1995).

Evaluación y validación de la estructura tridimensional de la PQQ- mGDH de Pseudomonas fluorescens

La validación de los modelos se evaluó con los programas BIOVIA ${ }^{\mathrm{TM}}$ (https://www.

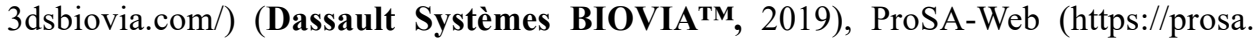


services.came.sbg.ac.at/prosa.php) y Verify 3D (http://nihserver.mbi.ucla.edu/Verify_3D/) (Lüthy, et al., 992). Todos los modelos generados por GENO3D se organizaron en el servidor Q-Mean (http://swissmodel.expasy.org/qmean/cgi/index.cgi) (Benkert, et al., 2009).

\section{Análisis de los dominios de la estructura secundaria de la PQQ- mGDH de Pseudomonas fluorescens}

La estructura secundaria de la PQQ- mGDH se predijo mediante la aplicación PSIPRED (http://bioinf.cs.ucl.ac.uk/psipred/) (McGuffin, et al., 2000), la cual utiliza un método muy preciso para la predicción de la estructura secundaria a partir de secuencias de aminoácidos.

\section{Determinación de los dominios proteicos}

Los dominios proteicos de la secuencia PQQ- mGDH en P. fluorescens se determinaron mediante la herramienta de búsqueda MOTIFsearch (https://www.genome.jp/tools/motif/).

\section{Resultados y discusión}

\section{Caracterización fisicoquímica de la enzima}

A partir de la secuencia proteica identificada para PQQ- mGDH de P. fluorescens (802aa) en la plataforma Expasy (https://web.expasy.org/compute_pi/), se determinó su peso molecular $(86.107,29 \mathrm{Da})$ y su punto isoeléctrico $(5,74)$, los cuales se acercan a los reportados por Cozier, et al. (1999), con pesos moleculares para esta molécula cercanos a los $8,7 \mathrm{kDa}$ y un punto isoeléctrico de 6,0 .

\section{Alineamiento por pares y múltiple}

La secuencia de la proteína correspondió a la referencia WP_014339995.1 del NCBI (https://www.ncbi.nlm.nih.gov/), es decir, la de la quinoproteína unida a membrana de la familia glucosa/quinato/shikimato deshidrogenasa [Pseudomonas fluorescens]. El alineamiento en BLAST permitió la identificación de múltiples especies de Pseudomonas, de las cuales se seleccionaron ocho que presentaban un valor de $\mathrm{E}$ de 0 , una identidad de $>88 \%$ y una cobertura de secuencia del $100 \%$. Las secuencias identificadas fueron: P. putida (WP_033044460.1), P. siryngae (WP_052966207.1), P. chlororaphis (WP_047737089.1), P. corrugata (WP_024780902.1), P. mediterranea (WP_047701274.1), P. frederiksbergensis (WP_039591463.1), P. brassicacearum (WP_153388849.1), P. kilonensis (WP_0460648 50.1), P. thivervalensis (AXA56945.1),

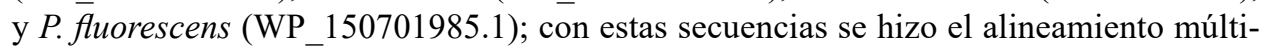
ple. El árbol filogenético (Figura 1) fue útil para asociar la relación de dichas especies mediante una representación gráfica en la cual se observa especialmente la agrupación entre $P$. putida y P. fluorescens. Con este porcentaje de identidad ( $>88 \%)$, el resultado del modelamiento podría extrapolarse a estas otras especies.

Modelamiento de la estructura tridimensional de la PQQ- mGDH de Pseudomonas fluorescens

Con base en los resultados del programa GENO3D para la secuencia de P. fluorescens, se estableció que la mejor secuencia para el modelamiento por homología correspondía a la quinohemoproteína alcohol deshidrogenasa (1kv9) en P. putida HK5 (Figura 2). Esta molécula presentó la mayor similitud con la secuencia PQQ-mGDH de $P$. fluorescens, con un valor de $\mathrm{E}$ de $1 \times 10^{-133}$ y una desviación media de 5,393140 ángstroms.

A partir de este análisis se generaron 10 modelos (Tabla 1) que se muestran superpuestos en la figura 3a, en la que se observa que la desviación entre los modelos es baja. En la figura 3b se observa el modelo más ajustado (modelo 10); además, se resaltan los ocho dominios del propulsor beta (triángulos punteados). En la figura 3c se muestra la molécula completa y se resaltan las estructuras secundarias de la arquitectura del dominio del propulsor beta, típico de este tipo de enzimas.

Los valores de correlación entre los diez modelos evaluados se determinaron mediante la desviación de la raíz cuadrada media (DRCM) con valores en ángstroms (Tabla 2). 


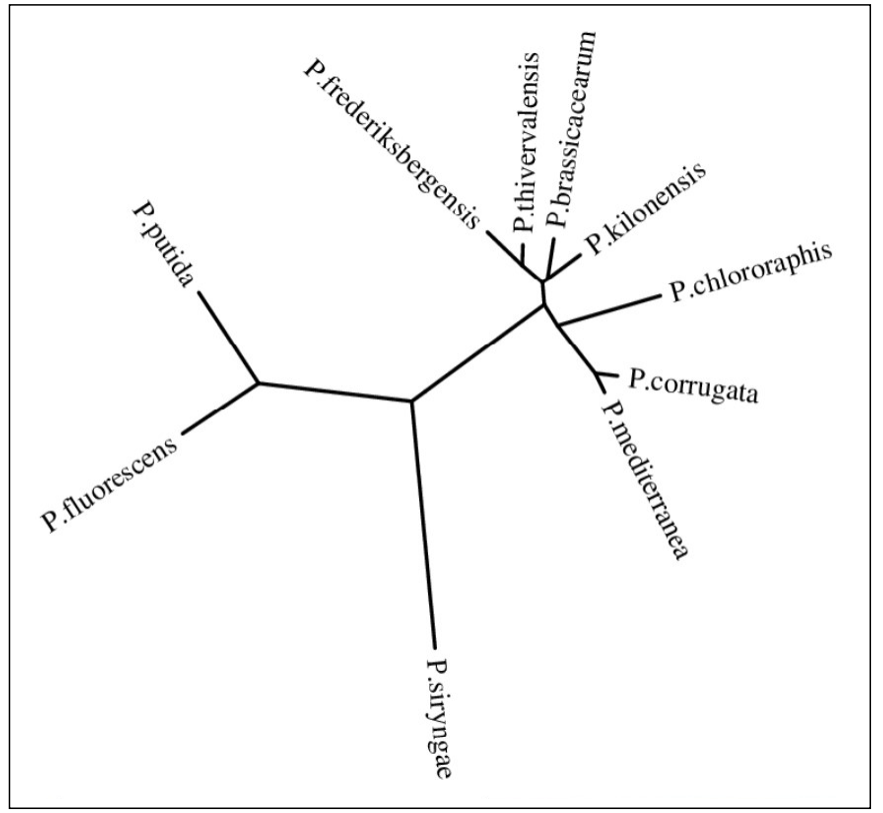

Figura 1. Árbol filogenético producto del alineamiento en Clustal omega de las secuencias de Pseudomonas reportadas para PQQ- mGDH utilizando los criterios descritos y a partir de los datos del alineamiento de Clustal Omega en la aplicación MABL (https://www.phylogeny.fr/)

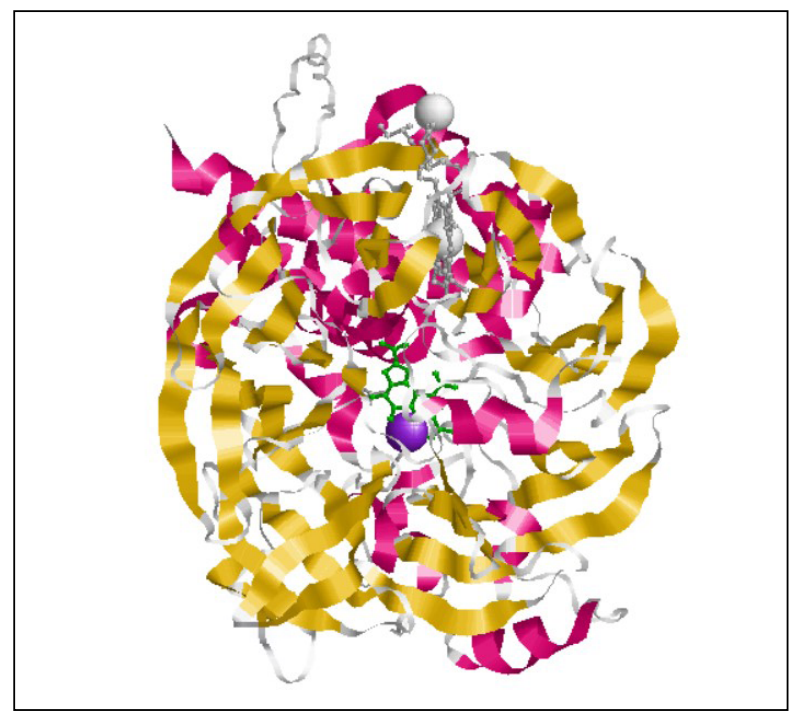

Figura 2. Motivo de unión al cofactor PQQ (propulsor beta). Se muestran los aminoácidos con los que interactúan: en verde se ve la molécula de PQQ en la quinohemoproteína alcohol deshidrogenasa en $P$. putida y en púrpura el HK5 unido a un átomo de calcio.

\section{Evaluación y validación de la estructura tridimensional de la PQQ- mGDH de Pseudomonas fluorescens}

Los resultados correspondientes al gráfico de Ramachandran, en el que se observan los aminoácidos presentes en la región de valores permitidos y no permitidos para las diferentes estructuras secundarias y la distribución de los valores Psi/Phi para el modelo de homología de la PQQ- mGDH de P. fluorescens obtenidos con el programa BIOVIA ${ }^{\mathrm{TM}}$ (Dassault Systèmes BIOVIA ${ }^{\text {TM }}$ 2019) (Figura 4). 
Tabla 1. Comparación de la calidad de los modelos producidos por el servidor Geno3D. Todos los modelos utilizaron la misma plantilla (1kv9). Las columnas representan los valores de energía en $\mathrm{Kcal} / \mathrm{mol}$, el porcentaje de residuos que se encuentra en la región nuclear y el de los residuos no permitidos en el diagrama de Ramachandran. Los valores de energía indican la fiabilidad y la precisión de la estructura.

\begin{tabular}{lcccc}
\hline $\begin{array}{l}\text { Nombre del } \\
\text { modelo }\end{array}$ & Molde & $\begin{array}{c}\text { Energía del } \\
\text { modelo Kcal/mol }\end{array}$ & $\begin{array}{c}\text { Porcentaje de residuos en la región del } \\
\text { núcleo del diagrama de Ramachandran }\end{array}$ & $\begin{array}{c}\text { Porcentaje de residuos no permitidos } \\
\text { en el diagrama de Ramachandran }\end{array}$ \\
\hline Modelo 1 & 1KV9 & $-24225,00$ & 54,4 & 5,6 \\
\hline Modelo 2 & - & $-24397,60$ & 53,8 & 6,3 \\
\hline Modelo 3 & - & $-24164,20$ & 49,6 & 4,6 \\
\hline Modelo 4 & - & $-24275,50$ & 53,8 & 5,6 \\
\hline Modelo 5 & - & $-24100,20$ & 54,8 & 6,2 \\
\hline Modelo 6 & - & $-24477,20$ & 46,3 & 4,8 \\
\hline Modelo 7 & - & $-23913,40$ & 50,8 & 4,0 \\
\hline Modelo 8 & - & $-14910,00$ & 30,8 & 8,1 \\
\hline Modelo 9 & - & $-24241,30$ & 51,5 & 5,6 \\
\hline Modelo 10 & - & $-24492,60$ & 55,6 & 5,0 \\
\hline
\end{tabular}
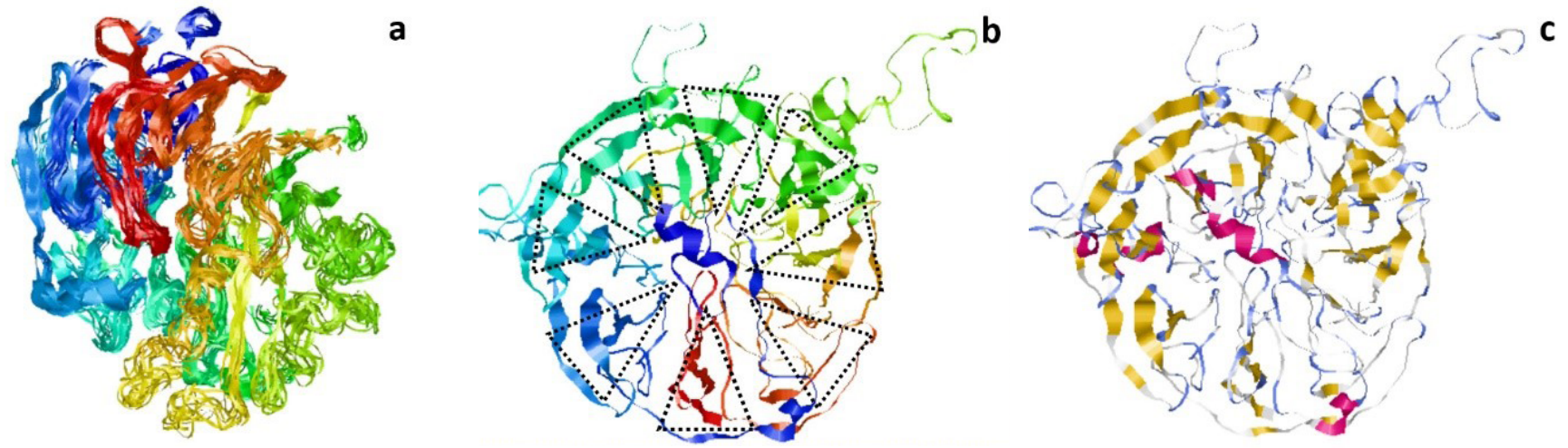

Figura 3. Modelos computacionales generados a partir del modelamiento por homología de la PQQ- mGDH en P. fluorescens. Se muestra en a) la superposición de los 10 modelos generados, en b) el modelo con los mejores valores (modelo 10) y en c) el detalle de las estructuras secundarias identificadas (color rosa: hélices alfa; color amarillo: hojas beta y en azul claro se muestran los plegamientos aleatorios (random coils)).

Tabla 2. Ajuste estructural entre los modelos (DRCM en ángstroms); desviación media: 5,393140A

\begin{tabular}{|c|c|c|c|c|c|c|c|c|c|c|}
\hline & Modelo 1 & Modelo 2 & Modelo 3 & Modelo 4 & Modelo 5 & Modelo 6 & Modelo 7 & Modelo 8 & Modelo 9 & Modelo 10 \\
\hline Modelo 1 & 0 & 1,36 & 1,39 & 1,38 & 1,52 & 1,41 & 1,61 & 20,88 & 1,39 & 1,48 \\
\hline Modelo 2 & 1,36 & 0 & 1,53 & 1,53 & 1,53 & 1,47 & 1,60 & 20,84 & 1,44 & 1,49 \\
\hline Modelo 3 & 1,39 & 1,53 & 0 & 1,50 & 1,45 & 1,38 & 1,56 & 20,88 & 1,44 & 1,53 \\
\hline Modelo 4 & 1,38 & 1,53 & 1,50 & 0 & 1,63 & 1,43 & 1,73 & 20,94 & 1,53 & 1,63 \\
\hline Modelo 5 & 1,52 & 1,53 & 1,45 & 1,63 & 0 & 1,44 & 1,56 & 21,08 & 1,47 & 1,60 \\
\hline Modelo 6 & 1,41 & 1,47 & 1,38 & 1,43 & 1,44 & 0 & 1,55 & 21,04 & 1,40 & 1,54 \\
\hline Modelo 7 & 1,61 & 1,60 & 1,56 & 1,73 & 1,56 & 1,55 & 0 & 21,02 & 1,54 & 1,49 \\
\hline Modelo 8 & 20,88 & 20,84 & 20,88 & 20,94 & 21,08 & 21,04 & 21,02 & 0 & 21,03 & 21,00 \\
\hline Modelo 9 & 1,39 & 1,44 & 1,44 & 1,53 & 1,47 & 1,40 & 1,54 & 21,03 & 0 & 1,47 \\
\hline Modelo 10 & 1,48 & 1,49 & 1,53 & 1,63 & 1,60 & 1,54 & 1,49 & 21,00 & 1,47 & 0 \\
\hline
\end{tabular}


El diagrama de Ramachandran (Ramachandran, et al., 1963) del modelo 10 de la PQQ- mGDH de P. fluorescens reveló que la mayor cantidad de residuos, $88,5 \%$, se localizó en el cuadrante superior izquierdo (región más favorable), que corresponde a las estructuras de hojas beta paralelas, hojas beta antiparalelas y giros; las estructuras secundarias sumaron un 55,6\% de los residuos totales (Figura 4). Además, se observa un cúmulo de residuos hacia el cuadrante inferior izquierdo que representa la región adicional permitida y en el que se encontraron los residuos principales que tienden a formar hélices alfa, los cuales sumaron un $30 \%$ del total (Figura 4). Este tipo de distribución de estructuras secundarias es típico del dominio propulsor beta, el cual es preponderante en este tipo de enzimas. El $5 \%$ de los aminoácidos totales se ubicó en las regiones no permitidas y, además, se observó que 520 residuos eran diferentes a la glicina-prolina; el número de glicinas, representado en los triángulos, fue de 67 , y el número de prolinas fue de 53 , para un total de 642 aminoácidos.

Con el fin de predecir la estructura secundaria propuesta para la secuencia de la PQQ- mGDH en $P$. fluorescens y confirmar los resultados observados en el diagrama de Ramachandran se utilizó la aplicación PSIPRED (UCL Department of Computer Science: Bioinformatics Group), análisis que evidenció que la enzima PQQ mGDH de P. fluorescens contiene 14 tramos de aminoácidos con tendencia a formar hélices alfa y 38 tramos de aminoácidos con tendencia a formar hojas beta. (Figura 2S, https://www.raccefyn.co/ index.php/raccefyn/article/view/1154/2924).

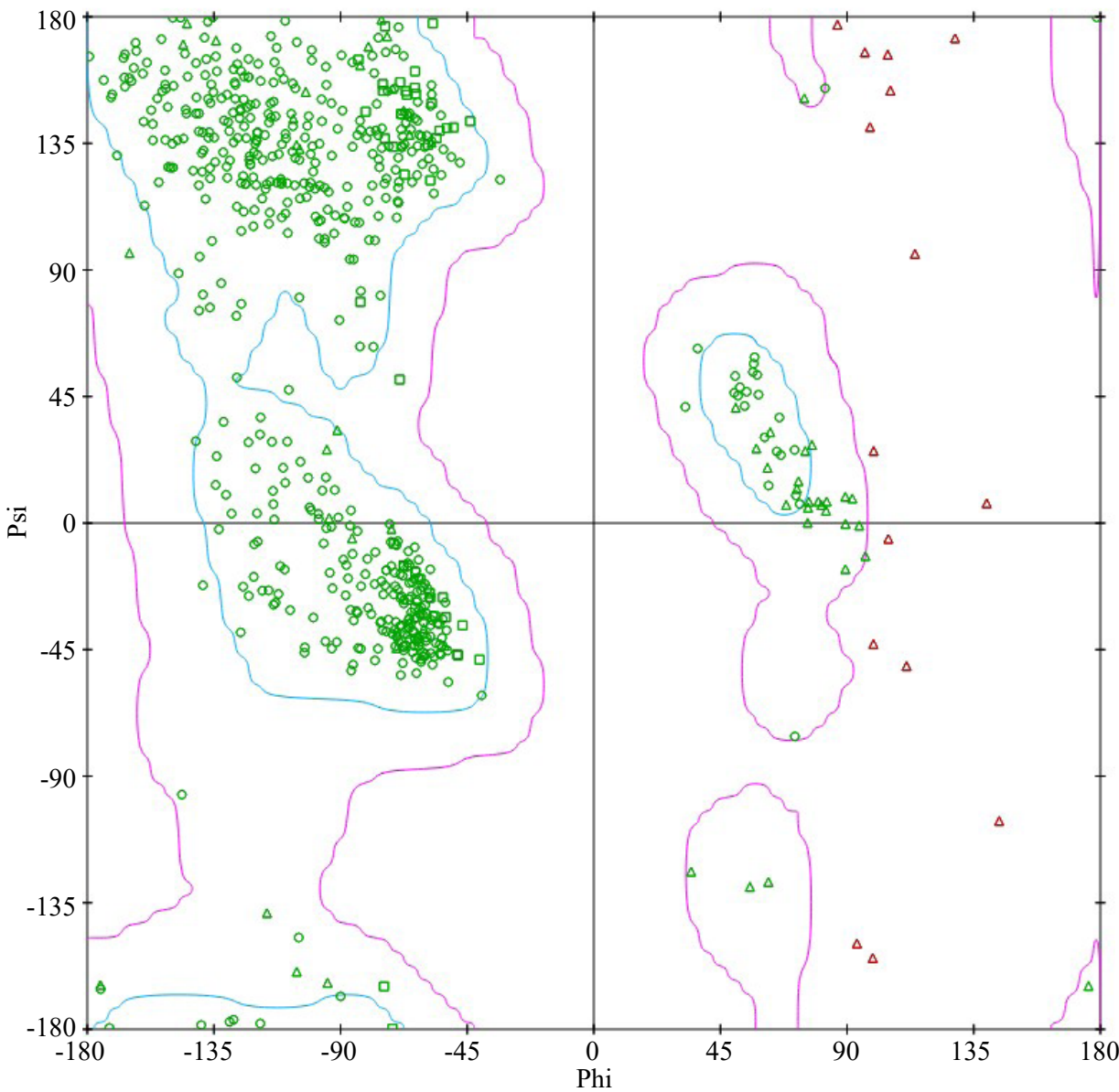

Figura 4. Diagrama de Ramachandran arrojado por el programa BIOVIA ${ }^{\mathrm{TM}}$ en el que se observa la presencia de cada uno de los aminoácidos de la secuencia en las regiones permitidas y no permitidas para las diferentes estructuras secundarias en la PQQ- mGDH de Pseudomonas fluorescens. 
Como parte del dominio preponderante de las PQQ- DH se conocen cinco clases de propulsores beta con ejes de pseudo-simetría de cuatro, cinco, seis, siete u ocho hojas beta (Pons, et al., 2012). En cuanto al propulsor beta de la PQQ mGDH en P. fluorescens, se han identificado ocho dominios de propulsores beta con cuatro hojas beta antiparalelas en cada uno de ellos (Pons, et al., 2012), y se ha establecido que las hojas primera y cuarta son casi perpendiculares entre sí (Kuriyan, et al., 2013) (Figura 3b). Debido a su estructura y plasticidad, las interacciones entre proteína y proteína que se forman son aquellas entre las caras superior, inferior, central y lateral del propulsor beta (Chen, et al., 2011). La función de la hélice puede variar según el número de hojas (Pons, et al., 2012), caso en el que los propulsores beta de cuatro hojas funcionan principalmente como proteínas de transporte $\mathrm{y}$, debido a su estructura, tienen una conformación favorable para la unión del sustrato (Chen, et al., 2011). A diferencia de los propulsores beta más grandes, el propulsor beta de cuatro hojas generalmente no puede hacer la catálisis por sí mismo, y requiere un cofactor para llevar a cabo dichas funciones. En el caso de la GDH, tanto la soluble como la que se une a la membrana, el cofactor utilizado es la pirroloquinolina quinona (PQQ).

En este modelamiento se estableció que la PQQ- mGDH de $P$. fluorescens presentaba cuatro hojas para cada uno de los dominios del propulsor beta, cuya estructura se estabiliza principalmente a través de interacciones hidrófobas de las láminas beta, en tanto que la estabilidad adicional puede provenir de enlaces de hidrógeno formados entre las láminas beta de los extremos $\mathrm{C}$ y N-terminales; además, se ha reportado la presencia de un enlace disulfuro (Pons, et al., 2012). Sin embargo, en este caso (modelo 10), la PQQ- mGDH de $P$. fluorescens no estableció, al parecer, un puente disulfuro, ya que las cisteínas más cercanas se encontraron a más de 6 ángstroms entre sí.

Con el fin de validar el modelo 10, se hizo un análisis de validación estructural utilizando la aplicación Prosa-Web (Wiederstein \& Sippl, 2007; Sippl, M.J., 1993). El puntaje $\mathrm{Z}$ para la calidad del modelo total fue de $-5,46$, el cual se encontró en el rango de las conformaciones nativas (Figura 5a). Además, para el análisis de la calidad local del modelo se midió la energía basada en conocimiento (Figura 5b) determinando la presencia de los picos correspondientes a las regiones de paso a través de la membrana de la proteína. Asimismo, se hizo un análisis con la herramienta Verify 3D, el cual confirmó los resultados
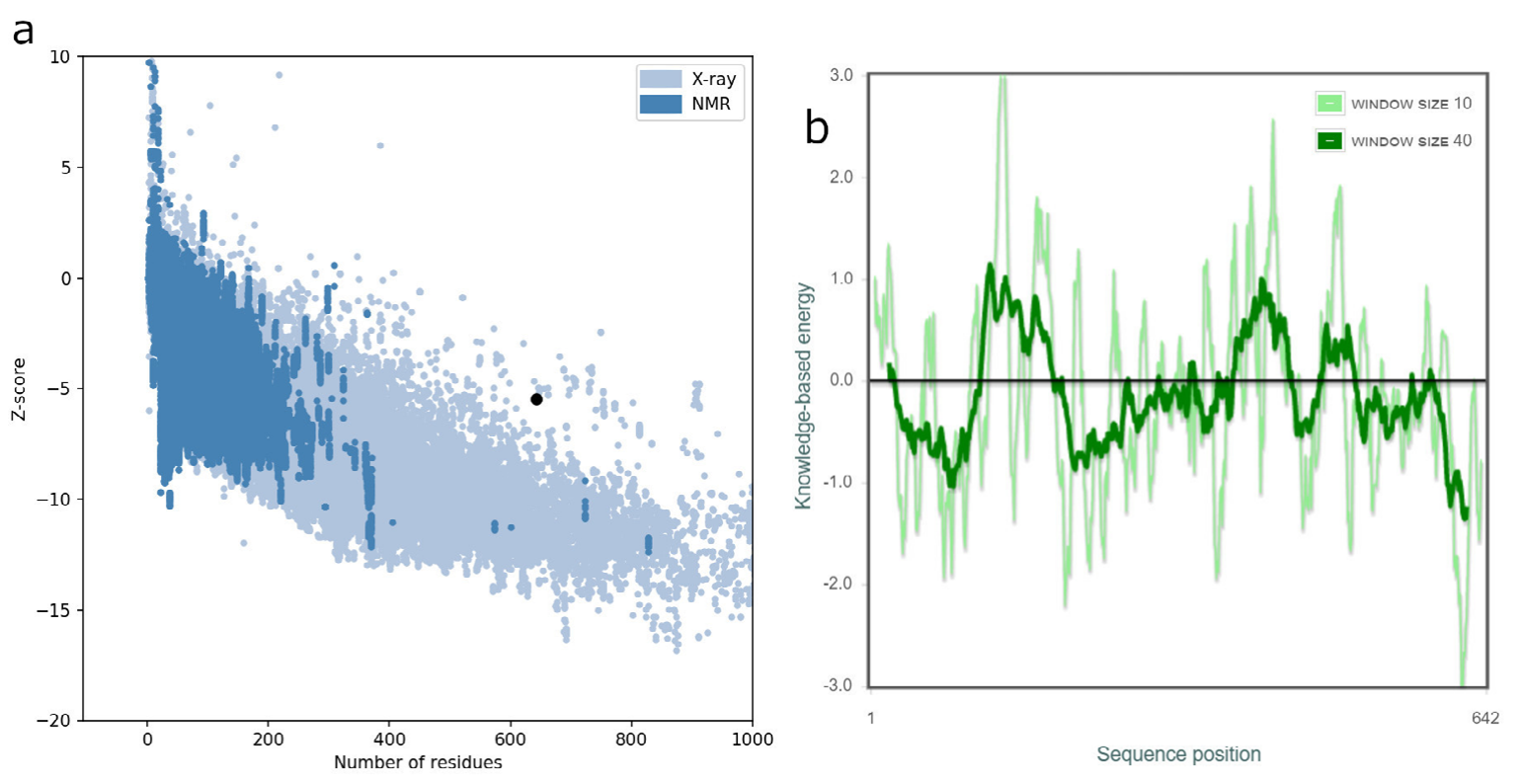

Figura 5. Análisis de validación de la estructura tridimensional del modelo propuesto (modelo 10) llevado a cabo en la aplicación Prosa-Web. a) Calidad total del modelo según el puntaje Z. b) Calidad local del modelo a través de la medición de la energía basada en conocimiento. 
obtenidos con la aplicación Prosa-web determinándose que el 85,36 \% de los aminoácidos tenía un valor mayor a 0,2 , con lo cual se verificó que la estructura era de alta calidad y había coherencia entre la secuencia y la estructura modelada (datos no mostrados), con lo cual quedó validado el modelo para la enzima PQQ- mGDH de P. fluorescens (Figura 6).

Dependiendo de su estabilidad, las PQQ mGDH pueden clasificarse como tipo I y tipo II. Las de tipo I se consideran de menor estabilidad que las de tipo II y han sido identificadas en E. coli y Pseudomonas sp, en tanto que las de tipo II se han encontrado en los géneros Acinetobacter y Gluconobacter (Anthony, 2001). En la medida en que el enlace disulfuro es una interacción que estabiliza estructuralmente las proteínas, la imposibilidad de que se forme este en la molécula modelada se podría asociar con el hecho de que las PQQ mGDH de P. fluorescens pertenecen al tipo I, de baja estabilidad.

\section{$P Q Q$ e interacciones moleculares con PQQ- $m G D H$}

La pirroloquinolina quinona (PQQ) es el grupo prostético de las quinoproteínas deshidrogenasas que catalizan la oxidación de alcoholes y azúcares aldosas en el periplasma de las bacterias Gram negativas, y se aisló primero de GDH y MDH (Anthony \& Zatman, 1967b; Hauge, 1964). Esta molécula es fácilmente liberada de las enzimas por desnaturalización; después de su purificación se demostró que era un compuesto ácido, altamente polar, con una fluorescencia verde muy característica (Anthony \& Zatman, 1967a). Posteriormente, su estructura fue dilucidada por cristalografía de rayos $\mathrm{X}$ y se la denominó metoxatina (Salisbury, et al., 1979). Su nombre completo es 2,7,9-tri-carboxi-1H-pirrol [2,3-f] quinolina-4,5-dione, y su nombre corto es pirroloquinolina quinona (PQQ) (Figura 3S, https://www.raccefyn.co/index.php/raccefyn/article/view/1154/2924).

$\mathrm{Su}$ química y su función en las deshidrogenasas fue demostrada por Duine (1991) y Duine \& Jongejan (1989), quienes la describieron en detalle y demostraron que una característica clave de su estructura es la orto-quinona en las posiciones $\mathrm{C} 4$ y $\mathrm{C} 5 \mathrm{del}$ anillo de quinolina, que se reduce a quinol durante la catálisis. La PQQ forma muy fácilmente aductos en la posición C5, que pueden ser mecanismos importantes en la medición e iden-

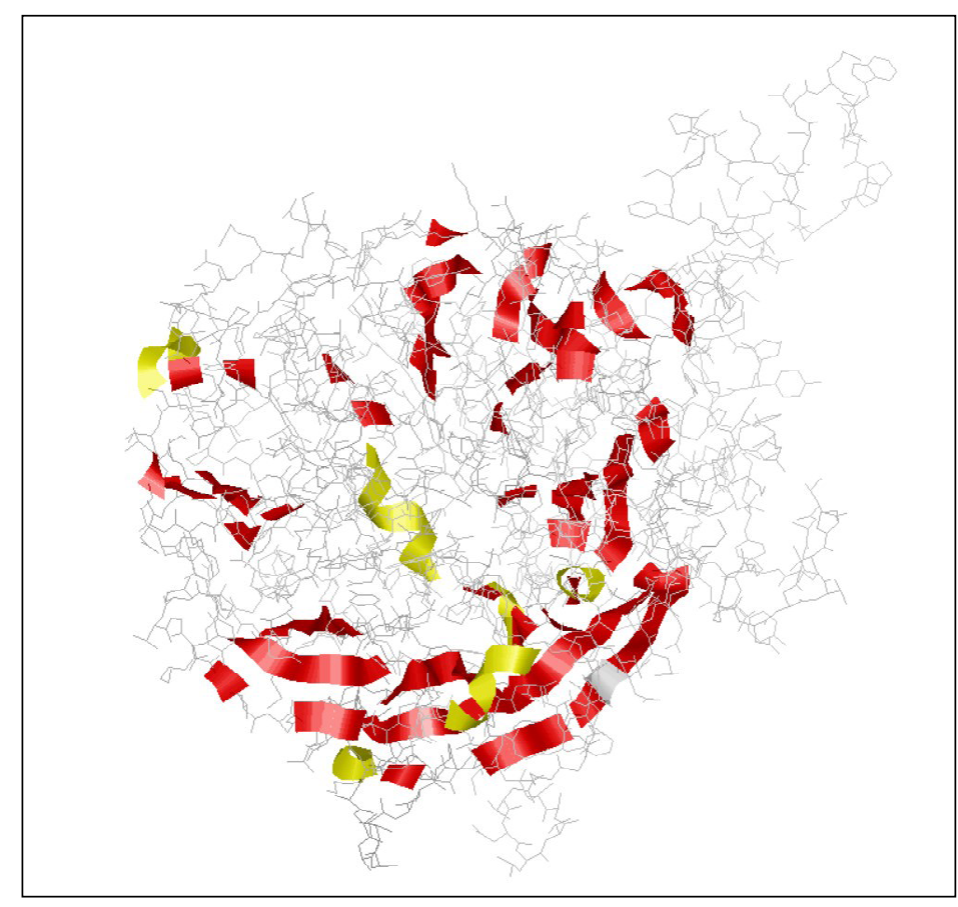

Figura 6. Modelo final propuesto para la enzima PQQ- mGDH de P. fluorescens. En rojo se muestran las hojas beta y en amarillo, las hélices que constituyen el dominio propulsor beta. 
tificación. El potencial redox de punto medio a un pH de 7 de la PQQ aislada es de aproximadamente $190 \mathrm{mV}$, pero es probable que esto se vea influenciado por su entorno en las deshidrogenasas. Una característica importante de la PQQ es su capacidad de acomplejar cationes divalentes en solución (Good, et al., 2016; Itoh, et al., 1998). Los compuestos modelo que los contienen pueden catalizar reacciones de oxidación (Itoh, et al., 1998; Itoh, et al., 2000), y hoy está claro que es probable que todas las enzimas que contienen PQQ tengan una acomplejada con un ion metálico divalente en su sitio activo.

En la aplicación del PDBsum (http://www.ebi.ac.uk/thornton-srv/databases/cgi-bin/ pdbsum/GetPage.pl?pdbcode=index.html) se identificaron los residuos que interaccionan con el cofactor PQQ para la molécula quinohemoproteína alcohol deshidrogenasa de $P$. putida utilizando con código de acceso $1 \mathrm{kv}$, entre los que se encuentran Glu (59), Arg (111), Thr (155), Gly (171), Ala (172), Thr (230), Lys (322), Asn (382), Trp (383), Val (525) y un átomo de Ca (802) (Figura 7).

Con el fin de determinar los aminoácidos que interaccionan con la PQQ en la molécula PQQ mGDH de P. fluorescens, se hizo un alineamiento en BLAST identificando las posiciones de los aminoácidos mostrados en la figura 7 y la figura 4S, https://www.raccefyn.co/ index.php/raccefyn/article/view/1154/2924. Al analizar las posiciones de los aminoácidos de unión a la PQQ para las dos secuencias de $P$. fluorescens y P. putida, se verificó que el 50 $\%$ de los aminoácidos (en verde en la Figura) fue idéntico en las dos secuencias (modelo/ molde; T-155 R-111, E-59 W-383, K-322), que un $20 \%$ (en azul) estaban conservados (modelo/molde: S/A-172, D/N-382), un $20 \%$ (en rojo) cambió totalmente (modelo/ molde: V/G-171, N/T-230) y un $10 \%$ no pudo ser identificado (Figura 5S, https://www. raccefyn.co/index.php/raccefyn/article/view/1154/2924). Estos resultados corroboraron cuáles eran los aminoácidos involucrados en la interacción con la molécula de PQQ y cuáles los cambios que se presentaban en $P$. fluorescens con referencia a $P$. putida.

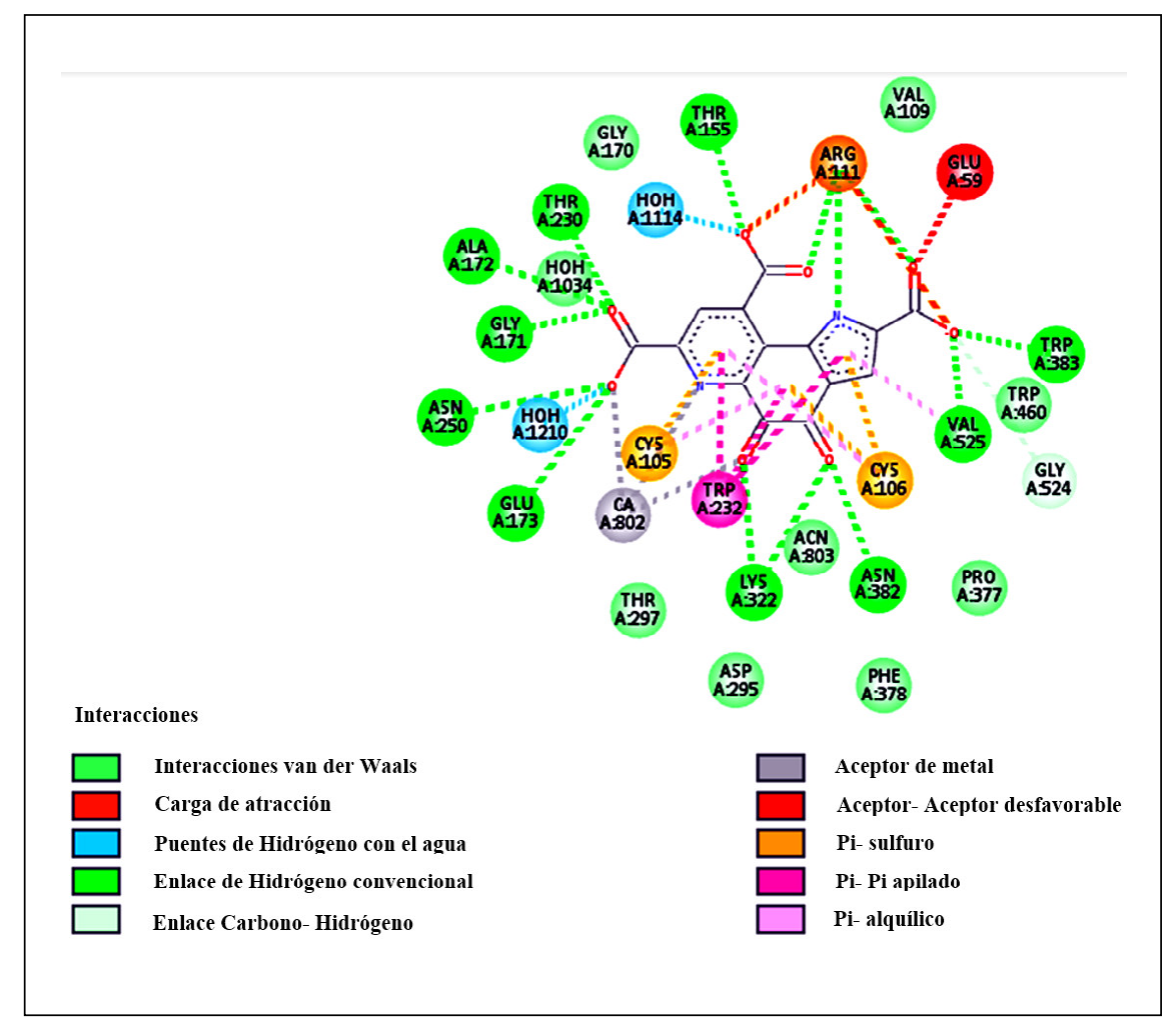

Figura 7. Interacciones de los aminoácidos con la PQQ. 
La búsqueda de los dominios se hizo en la plataforma MOTIF Search (https://www.genome. $\mathrm{jp} /$ tools/motif/) utilizando 0,85 como límite para el valor de E. Los resultados se muestran en la tabla 1S, https://www.raccefyn.co/index.php/raccefyn/article/view/1154/2924.

Es importante resaltar que se tomaron en cuenta los dominios de interés para el modelamiento de la enzima, pero que también se identificados algunos significativos de características generales de la proteína. Por ejemplo, la repetición del propulsor beta de la PQQ, que es uno de los más importantes y un tipo de arquitectura de proteínas con conformación beta caracterizada por cuatro a ocho láminas beta antiparalelas dobladas y dispuestas alrededor de un eje central. El sitio activo de la enzima se encuentra a menudo en la hendidura formada en el centro del propulsor por bucles que conectan los sucesivos dominios de cuatro láminas. En este caso, la repetición fue de una lámina beta (Pons, et al., 2012). Se identificaron también dominios directamente relacionados, como el PQQ, así como dominios de unión y de transporte a diversos monosacáridos, y dominios transmembrana. Asimismo, se determinaron algunas otras características en la molécula, como los dominios de holinas y el de transporte de aminoácidos, y resultó interesante encontrar dominios en proteínas que se activan en respuesta a las bajas temperaturas en las bacterias (Tabla 1S, https://www.raccefyn.co/index.php/raccefyn/article/view/1154/2924).

La caracterización estructural de esta molécula es importante porque su actividad es primordial en los procesos de solubilización del fosforo que hacen que $P$. fluorescens sea una importante promotora del crecimiento vegetal. Además de esta actividad promotora, las PQQ GDH se han aplicado en diversos campos, por ejemplo, para protocolos de análisis (Kim, et al., 2016), la producción de biocombustibles (Sakamoto, et al., 2015), y las aplicaciones biotecnológicas (Laurinavicius, et al., 2008) y de diagnóstico. También se ha estudiado la participación del grupo PQQ en el metabolismo del cristalino ocular y su relación con la formación de las cataratas inducidas con hidrocortisona en embriones de pollo y se ha establecido que este reprime su formación al inhibir las enzimas tirosinasa y aldosa reductasa implicadas en este padecimiento (Ameyama, et al., 1991; Hiraoka, et al., 1996; McIntire, 1998; Nishigori, et al., 1989).

\section{Conclusiones}

El modelamiento por homología es una herramienta in silico valiosa para la determinación de la estructura 3D de una proteína cuya estructura real no se ha obtenido, hasta donde se sabe, mediante difracción de rayos $\mathrm{X}$ ni resonancia magnética nuclear (RMN), por lo que constituye un primer paso hacia la investigación con métodos experimentales. Se sientan, además, las bases para el diseño de una metodología menos especulativa y útil para obtener datos sobre el funcionamiento de la proteína con fines biotecnológicos. Los resultados del análisis bioinformático de modelamiento por homología indicaron que la enzima PQQ- mGDH de $P$. fluorescens presenta homología con la enzima de P. putida, lo que sugiere que es un microorganismo promotor del crecimiento vegetal útil en los procesos de solubilización del fósforo disponible en el suelo y de aquellos minerales cuya solubilización requiera el ataque de ácidos orgánicos producidos por las bacterias, por ejemplo, la posterior asimilación del potasio por parte de la planta. Tales hipótesis computacionales proporcionan una plataforma para posteriores estudios sobre su potencial de promoción del crecimiento vegetal en microorganismos y sus mecanismos de acción.

\section{Información suplementaria}

Ver la información suplementaria en https://www.raccefyn.co/index.php/raccefyn/article/ view/1154/2924

\section{Contribución de los autores}

Todos los autores participaron activamente en la búsqueda y análisis de los datos y en la discusión de los resultados, así como la validación de la información para la preparación y revisión del manuscrito. 


\section{Conflicto de intereses}

Los autores manifiestan que no existe ningún tipo de conflicto de intereses con respecto a los resultados de la presente investigación.

\section{Referencias}

Alori, E. T., Glick, B. R., \& Babalola, O. O. (2017). Microbial phosphorus solubilization and its potential for use in sustainable agriculture. Frontiers in Microbiology. 8: 971. Doi: 10.3389/ fmicb.2017.00971

Ameyama, M., Matsushita, K., Shinagawa, E., \& Adachi, O. (1991). Biochemical and physiological functions of pyrroloquinoline quinone. Vitamins and Hormones. 46: 229-270. http:// www.ncbi.nlm.nih.gov/pubmed/1660640

An, R., \& Moe, L. A. (2016). Regulation of Pyrroloquinoline Quinone-Dependent Glucose Dehydrogenase Activity in the Model Rhizosphere-Dwelling Bacterium Pseudomonas putida KT2440. Applied and Environmental Microbiology. 82 (16): 4955-4964. Doi: 10.1128/aem.00813-16

Anthony, C. (2001). Pyrroloquinoline quinone (PQQ) and quinoprotein enzymes. Antioxidants and Redox Signaling. 3 (5): 757-774. Doi: 10.1089/15230860152664966

Anthony, C., \& Zatman, L. (1967a). The microbial oxidation of methanol. The prosthetic group of the alcohol dehydrogenase of Pseudomonas sp. M27: a new oxidoreductase prosthetic group. Biochemical Journal. 104 (3): 960-969. Doi: 10.1042/bj1040960

Anthony, C., \& Zatman, L. J. (1967b). The microbial oxidation of methanol. The prosthetic group of the alcohol dehydrogenase of Pseudomonas sp. M27: a new oxidoreductase prosthetic group. The Biochemical Journal. 104 (3): 960-969. Doi: 10.1042/bj1040960

Asahi, Y., Noiri, Y., Igarashi, J., Asai, H., Suga, H., Ebisu, S. (2010). Effects of N-acyl homoserine lactone analogues on Porphyromonas gingivalis biofilm formation. Journal of Periodontal Research. 45 (2): 255-261. Doi: 10.1111/j.1600-0765.2009.01228.x

Babnigg, G., \& Joachimiak, A. (2010). Predicting protein crystallization propensity from protein sequence. Journal of Structural and Functional Genomics. 11 (1): 71-80. Doi: 10.1007/ s10969-010-9080-0

Bailey, T. L., Boden, M., Buske, F. A., Frith, M., Grant, C. E., Clementi, L., Noble, W. S. (2009). MEME SUITE: tools for motif discovery and searching. Nucleic Acids Research. 37: W202-W208. Doi: 10.1093/nar/gkp335

Ben Farhat, M., Fourati, A., Chouayekh, H. (2013). Coexpression of the pyrroloquinoline quinone and glucose dehydrogenase genes from Serratia marcescens CTM 50650 conferred high mineral phosphate-solubilizing ability to Escherichia coli. Applied Biochemistry and Biotechnology. 170 (7): 1738-1750. Doi: 10.1007/s12010-013-0305-0

Benkert, P., Künzli, M., Schwede, T. (2009). QMEAN server for protein model quality estimation. Nucleic Acids Research: 37 (suppl_2): W510-W514. Doi: 10.1093/nar/gkp322

Chapman, H. N., Fromme, P., Barty, A., White, T. a, Kirian, R. a, Aquila, A., Spence, J. C. H. (2011). Femtosecond X-ray protein nanocrystallography - Supplementary Information. Nature. 470 (7332): 73-77. Doi: 10.1038/nature09750

Chen, C. K.-M., Chan, N.-L., Wang, A. H.-J. (2011). The many blades of the $\beta$-propeller proteins: conserved but versatile. Trends in Biochemical Sciences. 36 (10): 553-561. Doi: 10.1016/j. tibs.2011.07.004

Combet, C., Jambon, M., Deleage, G., Geourjon, C. (2002). Geno3D: automatic comparative molecular modelling of protein. Bioinformatics. 18 (1): 213-214. Doi: 10.1093/ bioinformatics/18.1.213

Cozier, G. E., Salleh, R. A., Anthony, C. (1999). Characterization of the membrane quinoprotein glucose dehydrogenase from Escherichia coli and characterization of a site-directed mutant in which histidine-262 has been changed to tyrosine. The Biochemical Journal. 340 (Pt 3): 639-647. http://www.ncbi.nlm.nih.gov/pubmed/10359647

Dassault Systèmes BIOVIA. (2019). Discovery studio. San Diego: Dessault Systemes.

Duine, J. A. (1991). Quinoproteins: enzymes containing the quinonoid cofactor pyrroloquinoline quinone, topaquinone or tryptophan-tryptophan quinone. European Journal of Biochemistry. 200 (2): 271-284. Doi: 10.1111/j.1432-1033.1991.tb16183.x

Duine, J. A. \& Jongejan, J. A. (1989). Quinoproteins, enzymes with pyrrolo-quinoline quinone as cofactor. Annual Review of Biochemistry. 58: 403-426. Doi: 10.1146/annurev.bi.58. 070189.002155 
Goldstein, A., Lester, T., Brown, J. (2003). Research on the metabolic engineering of the direct oxidation pathway for extraction of phosphate from ore has generated preliminary evidence for PQQ biosynthesis in Escherichia coli as well as a possible role for the highly conserved region of quinoprote. Biochimica et Biophysica Acta - Proteins and Proteomics. 1647 (1-2): 266-271. Doi: 10.1016/S1570-9639(03)00067-0

Good, N. M., Vu, H. N., Suriano, C. J., Subuyuj, G. A., Skovran, E., Martínez-Gómez, N. C. (2016). Pyrroloquinoline quinone ethanol dehydrogenase in Methylobacterium extorquens AM1 extends lanthanide-dependent metabolism to multicarbon substrates. Journal of Bacteriology. 198 (22): 3109-3118. Doi: 10.1128/JB.00478-16

Hamilton, N. \& Huber, T. (2008). An introduction to protein contact prediction. Methods in Molecular Biology (Clifton, N.J.). 453: 87-104. Doi: 10.1007/978-1-60327-429-6_3

Hauge, J. G. (1964). Glucose Dehydrogenase of Bacterium with a Novel Prosthetic Group * an Enzyme. The Journal of Biological Chemistry. 239 (11): 3630-3639. http://www.jbc.org/

Hiraoka, T., Clark, J. I., LI, X. Y., Thurston, G. M. (1996). Effect of selected anti-cataract agents on opacification in the selenite cataract model. Experimental Eye Research: 62 (1): 11-19. Doi: 10.1006/exer.1996.0002

Itoh, S, Kawakami, H., Fukuzumi, S. (1998). Model studies on calcium-containing quinoprotein alcohol dehydrogenases. Catalytic role of $\mathrm{Ca} 2+$ for the oxidation of alcohols by coenzyme PQQ (4,5-dihydro-4,5-dioxo-1H-pyrrolo[2,3-f]quinoline-2, 7,9-tricarboxylic acid). Biochemistry. 37 (18): 6562-6571. Doi: 10.1021/bi9800092

Itoh, Shinobu, Kawakami, H., Fukuzumi, S. (2000). Development of the active site model for calcium-containing quinoprotein alcohol dehydrogenases. Journal of Molecular Catalysis - B Enzymatic. 8 (1-3): 85-94. Doi: 10.1016/S1381-1177(99)00070-3

Kim, H.-W., Wang, J.-Y., Lee, J.-Y., Park, A.-K., Park, H., Jeon, S.-J. (2016). Biochemical and structural characterization of quinoprotein aldose sugar dehydrogenase from Thermus thermophilus HJ6: Mutational analysis of Tyr156 in the substrate-binding site. Archives of Biochemistry and Biophysics. 608: 20-26. Doi: 10.1016/j.abb.2016.08.022

Krishnaraj, P. U. \& Goldstein, A. (2002). Cloning of a Serratia marcescens DNA fragment that induces quinoprotein glucose dehydrogenase-mediated gluconic acid production in Escherichia coli in the presence of stationary phase Serratia marcescens. FEMS Microbiology Letters. 205 (2): 215-220. Doi: 10.1016/s0378-1097(01)00472-4

Kuriyan, J., Konforti, B., Wemmer, D. (2013). The molecules of life: physical and chemical principles. Garland Science. 1032 p. New York.

Laurinavicius, V., Kurtinaitiene, B., Stankeviciute, R. (2008). Behavior of PQQ glucose dehydrogenase on Prussian blue-modified carbon electrode. Electroanalysis. 20 (13): 1391-1395. Doi: 10.1002/elan.200804216

Lidbury, I. D. E. A., Murphy, A. R. J., Scanlan, D. J., Bending, G. D., Jones, A. M. E., Moore, J. D., Wellington, E. M. H. (2016). Comparative genomic, proteomic and exoproteomic analyses of three Pseudomonas strains reveals novel insights into the phosphorus scavenging capabilities of soil bacteria. Environmental Microbiology. 18 (10): 3535-3549. Doi: 10.1111/1462-2920.13390

Lüthy, R., Bowie, J. U., Eisenberg, D. (1992). Assessment of protein models with three-dimensional profiles. Nature. 356 (6364): 83-85. Doi: 10.1038/356083a0

Madeira, F., Park, Y. mi, Lee, J., Buso, N., Gur, T., Madhusoodanan, N., López, R. (2019). The EMBL-EBI search and sequence analysis tools APIs in 2019. Nucleic Acids Research. 47 (W1): W636-W641. Doi: 10.1093/nar/gkz268

McGuffin, L. J., Bryson, K., Jones, D. T. (2000). The PSIPRED protein structure prediction server. Bioinformatics. 16 (4): 404-405. Doi: 10.1093/bioinformatics/16.4.404

McIntire, W. S. (1998). Newly discovered redox cofactors: possible nutritional, medical, and pharmacological relevance to higher animals. Annual Review of Nutrition. 18: 145-177. Doi: 10.1146/annurev.nutr.18.1.145

Nishigori, H., Yasunaga, M., Mizumura, M., Lee, J. W., Iwatsuru, M. (1989). Preventive effects of pyrroloquinoline quinone on formation of cataract and decline of lenticular and hepatic glutathione of developing chick embryo after glucocorticoid treatment. Life Sciences. 45 (7): 593-598. Doi: 10.1016/0024-3205(89)90044-1

Pérez, S., Coto, O., Echemendía, M., Ávila, G. (2015). Pseudomonas fluorescens Migula, ¿control biológico o patógeno? Rev. Protección Veg. 30 (3): 225-234.

Pons, T., Gómez, R., Chinea, G., Valencia, A. (2012). Beta-propellers: Associated Functions and their Role in Human Diseases. Current Medicinal Chemistry. 10 (6): 505-524. Doi: 10.2174/0929867033368204 
Ramachandran, G. N., Ramakrishnan, C., Sasisekharan, V. (1963). Stereochemistry of polypeptide chain configurations. Journal of Molecular Biology. 7: 95-99. Doi: 10.1016/S00222836(63)80023-6

Sakamoto, H., Uchii, T., Yamaguchi, K., Koto, A., Takamura, E.-I., Satomura, T., ... Suye, S.-I. (2015). Construction of a biocathode using the multicopper oxidase from the hyperthermophilic archaeon, Pyrobaculum aerophilum: towards a long-life biobattery. Biotechnology Letters. 37 (7): 1399-1404. Doi: 10.1007/s10529-015-1819-z

Salisbury, S. A., Forrest, H. S., Cruse, W. B. T., Kennard, O. (1979). A novel coenzyme from bacterial primary alcohol dehydrogenases [13]. Nature. 280: 843-844. Doi: 10.1038/280843a0

Sánchez-Garcés, M. A., Álvarez-Camino, J. C., Corral-Pavón, E., González-Martínez, R., Alves-Marques, J., Párraga-Manzol, G., Gay-Escoda, C. (2012). Revisión bibliográfica de Implantología Bucofacial del año 2010: Segunda parte. Avances En Periodoncia e Implantología Oral. 24 (2): 77-94. Doi: 10.4321/s1699-65852012000200003

Sayle, R. (1995). RASMOL: biomolecular graphics for all. Trends in Biochemical Sciences. 20 (9): 374-376. Doi: 10.1016/S0968-0004(00)89080-5

Sippl, M. J. (1993). Recognition of errors in three-dimensional structures of proteins. Proteins: Structure, Function, and Genetics. 17 (4): 355-362. Doi: 10.1002/prot.340170404

Takeda, K., Ishida, T., Yoshida, M., Samejima, M., Ohno, H., Igarashi, K., Nakamura, N. (2019). Crystal Structure of the Catalytic and Cytochrome b Domains in a Eukaryotic Pyrroloquinoline Quinone-Dependent Dehydrogenase. Applied and Environmental Microbiology. 85 (24): 1-13. Doi: 10.1128/AEM.01692-19

Vera-Cardoso, B. C., Muñoz-Rojas, J., Munive, J. A., Marín-Cevada, V., Flores-Encarnación, M., \& Ricardo, C. (2017). Pirroloquinolinaquinona (PQQ) y las bacterias promotoras del crecimiento vegetal (PGPR). De la biosíntesis a los fenotipos. Alianzas \& Tendencias. 2 (1): 22-29.

Wen, Z. T., Nguyen, A. H., Bitoun, J. P., Abranches, J., Baker, H. V, \& Burne, R. A. (2011). Transcriptome analysis of LuxS-deficient Streptococcus mutans grown in biofilms. Molecular Oral Microbiology. 26 (1): 2-18. Doi: 10.1111/j.2041-1014.2010.00581.x

Wiederstein, M., \& Sippl, M. J. (2007). ProSA-web: interactive web service for the recognition of errors in three-dimensional structures of proteins. Nucleic Acids Research. 35: 407-410. Doi: $10.1093 / \mathrm{nar} / \mathrm{gkm} 290$

Yamamoto, M., Hirata, K., Hikima, T., Kawano, Y., Ueno, G. (2010). Protein micro-crystallography with a new micro-beam beamline. Yakugaku Zasshi : Journal of the Pharmaceutical Society of Japan. 130 (5): 641-648. Doi: 10.1248/yakushi.130.641

Zdobnov, E. M. \& Apweiler, R. (2001). InterProScan - An integration platform for the signaturerecognition methods in InterPro. Bioinformatics. 17 (9): 847-848. Doi: 10.1093/ bioinformatics/17.9.847 